\title{
Synthesis and Emission Behavior of 1,3-diarylisobenzofuran-5,6-dicarboximides and Their Transformation into Naphthalene-2,3:6,7-bis(dicarboximide)s
}

\author{
Haruki Shimosasa ${ }^{1}$, Ryuta Miyatake ${ }^{2}$, Naoki Kobayashi ${ }^{1}$, Mitsunori Oda ${ }^{1}$, \\ ${ }^{1}$ Department of Chemistry, Faculty of Science, Shinshu University, Nagano, Japan \\ ${ }^{2}$ Centre for Environmental Conservation and Research Safety, University of Toyama, Toyama, Japan
}

Email address:

mituoda@shinshu-u.ac.jp (M. Oda)

${ }^{*}$ Corresponding author

\section{To cite this article:}

Haruki Shimosasa, Ryuta Miyatake, Naoki Kobayashi, Mitsunori Oda. Synthesis and Emission Behavior of 1,3-diarylisobenzofuran-5,6-dicarboximides and Their Transformation into Naphthalene-2,3:6,7-bis(dicarboximide)s. Modern Chemistry. Vol. 4, No. 2, 2016 pp. 16-23. doi: 10.11648/j.mc.20160402.11

Received: March 10, 2016; Accepted: March 18, 2016; Published: April 6, 2016

\begin{abstract}
Phosphine-assisted annulation of 2,5-diarylfuran-3,4-dicarbaldehydes with maleimides provided the title isobenzofurans in satisfactory yields. An effect of the substituents at the para position of the aryl groups in these isobenzofurans was demonstrated clearly by a red shift in their UV-vis absorption and emission spectra. They were transformed into the corresponding naphthalene-2,3:6,7-bis(dicarboximide)s by Diels-Alder reaction with another maleimide and subsequent dehydration with the aid of trifluoromethanesulfonic acid. Emission behavior of the title bis(dicarboximide)s is also described.
\end{abstract}

Keywords: Isobenzofuran, Naphthalene-bis(dicarboximide)s, Furan-3,4-dicarbaldehydes, Substituent Effect, Dehydration, Diels-Alder Reaction

\section{Introduction}

Arene-dicarboximides show interesting photochemical and photophysical properties, such as photoreduction [1, 2] photocycloaddition [3, 4], and photo-excited emission [5-8]. Heagy et al. reported that dual fluorescent naphthalene-2,3dicaboximides could be used in white organic light-emitting devices (OLEDs) [9]. Also, we reported synthesis of various 1,6-methano [10] annulene-3,4-dicaboximides and their emission behavior [10]. The results indicate that arene-dicarboximides are promising agents for electronic materials. Arene-dicarboximides can be obtained by several simple ways, such as condensation of anhydride with amine $[11,13-15]$ and Gabriel reaction of $N$-unsubstituted dicarboximide with alkyl or aryl haide [16-18]. Besides, an approach by Diels-Alder reaction via arene-o-quinodimethane or isobenzofuran constructing concomitantly another benzene ring has been reliable [19-22]. In 1979, Haddadin et al. reported an interesting synthetic method, in which $o$-phthaldehyde (1) reacts with $N$-phenylmaleimide (3) in the presence of triethylphosphite to give $N$-phenylnaphthalene-2,3-dicarboximide (2) in a satisfactory yield $(62 \%)$ [23]. However, application to synthesis of anthracene- 2,3-dicarboximide from 2,3-naphthaldehyde (4) by this method resulted in a low yield (35\%) of the product 5 . Notwithstanding the fascinating methodology based on the result of the Haddadin et al., there had been no work of its improvement reported for a long time. We reported the improved method for the Haddadin's annulation, that is, we found that various naphthalene- and anthracene-dicarboximides were obtained in good yields under the conditions with trioctylphosphine, instead of triethylphosphite, in re- fluxing dioxane (Scheme 1) [24]. We have applied our improved method to synthesize the title heterocyclic com pounds. In this paper, we describe the synthesis of the 1,3-diarylisobenzofuran-5,6-dicarboximides (6-8) and their 
transformation to naphthalene-bis(dicarboximide)s.

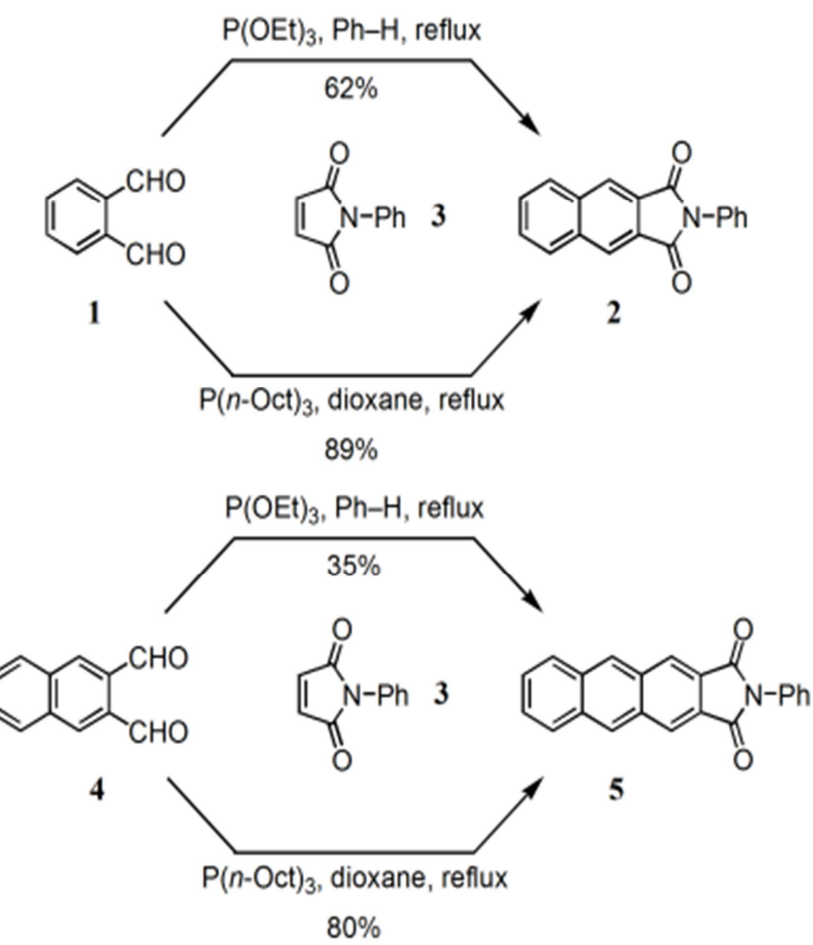

Scheme 1. Synthetic methods for arene-dicarboximides 2 and 5.

\section{Experimental}

\subsection{General Remarks}

Melting points were measured on a Yanaco MP-3 and are uncorrected. IR spectra were recorded on a JASCO FT/IR-4100 spectrometer. UV-vis spectra were recorded on a Shimadzu UV-2550 spectrometer. Emission spectra were recorded on a Shimadzu RF5300-PC spectrometer. Emis-sion quantum yields were obtained by comparison with that of anthracene ( $\Phi=27 \%$ in ethanol). ${ }^{1} \mathrm{H}$ - and ${ }^{13} \mathrm{C}$-NMR spectra were recorded on JEOL $\lambda 400$ and ECA500 spectrometers. Chemical shift values of tetramethylsilane ( $\delta=0 \mathrm{ppm}$ ) for ${ }^{1} \mathrm{H}-\mathrm{NMR}$ spectra and $\mathrm{CDCl}_{3}$ ( $\delta=77.0 \mathrm{ppm}$ ) for ${ }^{13} \mathrm{C}$-NMR spectra were used as internal standard. Mass spectra were measured on a JMS-700 mass spectrometer. Column chromatography was performed with silica gel $60 \mathrm{~N}$ from Kanto Chem. Dioxane, dimethylsulfoxide, tetrahydrofuran (THF) and triethylamine were purchased from Kanto Chem. and were distilled over $\mathrm{CaH}_{2}$. Dichloromethane (DCM), chloroform, dichloroethane (DCE), and acetonitrile were also purchased from Kanto Chem. and were distilled over $\mathrm{P}_{2} \mathrm{O}_{5}$. Trioctylphosphine, $\quad \mathrm{N}$-methylmaleimide, $N$-ethylmaleimide,

$N$-cyclohexylmaleimide, $N$-phenylmaleimide, and trifluoromethanesuifonic acid were purchased from Tokyo Chemical Industry, Inc. Oxalylchloride, $\mathrm{LiAlH}_{4}$, and thionylchloride were purchased from Wako Chem. $N$-p-Anisylmaleimide was prepared according to a two-step procedure from maleic anhydride and $p$-anisidine reported by Cava et al. [30-31] 2,5-Diarylfuran-3,4-dicarbaldehydes were prepared according to a procedure reported by Wang et al. [25]

\subsection{General Procedure for Synthesis of 2,5-diarylfuran-3,4-dicarbaldehydes}

A solution of 2,5-diaryl-3,4-bis(methoxycarbonyl)furan (19.0 mmol) in $70 \mathrm{~mL}$ of THF was added slowly to a suspension of $1.14 \mathrm{~g}$ of $\mathrm{LiAlH}_{4}(30.0 \mathrm{mmol})$ in $50 \mathrm{~mL}$ of THF at $0^{\circ} \mathrm{C}$. After being stirred at room temperature for $30 \mathrm{~h}$, the reaction mixture was carefully quenched by ethanol/water. The resulted mixture was passed through a Celite pad and was washed well with ether. The filtrate was dried over $\mathrm{Na}_{2} \mathrm{SO}_{4}$ and the solvent was evaporated to give the crude corresponding diol, which was used without further purification in the next Swern oxidation.

A solution of dimethylsulfoxide $(2.8 \mathrm{~mL})$ in $7 \mathrm{~mL}$ of $\mathrm{DCM}$ was added dropwise to a solution of oxalylchloride $(1.50 \mathrm{~mL}$, $17.7 \mathrm{mmol})$ in $40 \mathrm{~mL}$ of $\mathrm{DCM}$ at $-80^{\circ} \mathrm{C}$, followed by addition of a solution of the diol $(5.90 \mathrm{mmol})$ in $5 \mathrm{~mL}$ of DMSO and 15 $\mathrm{mL}$ of DCM, and then triethylamine $(11 \mathrm{~mL})$. After being stirred at the same temperature for $3 \mathrm{~h}$, the resulted reaction mixture was poured into water $(100 \mathrm{~mL})$ and was extracted with DCM (30 mL x 3). The combined organic layer was washed with brine and dried over $\mathrm{Na}_{2} \mathrm{SO}_{4}$. The solvent was removed and the residual solids were purified by recrystallization from hexane/DCM to give the dialdehyde.

9 (75\% from 12): Colorless needles, m.p. $137-138^{\circ} \mathrm{C}$ (lit. [32] $\left.126-128^{\circ} \mathrm{C}\right) .{ }^{1} \mathrm{HNMR}\left(500 \mathrm{MHz}, \mathrm{CDCl}_{3}\right) \delta=10.44(\mathrm{~s}$, $2 \mathrm{H}), 7.98(\mathrm{~m}, 4 \mathrm{H}), 754(\mathrm{~m}, 6 \mathrm{H}) \mathrm{ppm} ;{ }^{13} \mathrm{CNMR}\left(\mathrm{CDCl}_{3}, 125\right.$ MHz) $\delta=186.8,159.4,131.0,128.9,128.6,127.9,121.4$ ppm; IR (KBr) $v=1686(\mathrm{vs}), 1671$ (vs) $\mathrm{cm}^{-1}$.

10 (77\% from 13): Yellowish powder, m.p. $137.5-138.5^{\circ} \mathrm{C}$. ${ }^{1} \mathrm{HNMR}\left(\mathrm{CDCl}_{3}, 500 \mathrm{MHz}\right) \delta=10.4(\mathrm{~s}, 2 \mathrm{H}), 7.86(\mathrm{~d}, J=8.3 \mathrm{~Hz}$, $4 \mathrm{H}), 7.33(\mathrm{~d}, J=8.3 \mathrm{~Hz}, 4 \mathrm{H}), 2.45(\mathrm{~s}, 6 \mathrm{H}) \mathrm{ppm} ;{ }^{13} \mathrm{CNMR}$ $\left(\mathrm{CDCl}_{3}, 126 \mathrm{MHz}\right) \delta=186.9,159.6,141.5,129.6,128.5,125.2$, 120.9, 21.6 ppm; IR (KBr) $v=1685$ (vs), $1677(\mathrm{~s}), \mathrm{cm}^{-1}$; MS $m / z(\%)=304\left(\mathrm{M}^{+}, 100\right), 276(46), 261$ (13), 119 (42), 91 (23), 65 (6). HRMS calcd for $\mathrm{C}_{20} \mathrm{H}_{16} \mathrm{O}_{3}\left(\mathrm{M}^{+}\right)$304.1099, found 304.1106 .

11 (79\% from 14): Yellow powder, m.p. $147-148^{\circ} \mathrm{C}$. ${ }^{1} \mathrm{HNMR}\left(500 \mathrm{MHz}, \mathrm{CDCl}_{3}\right) \delta=10.41(\mathrm{~s}, 2 \mathrm{H}), 7.95(\mathrm{dt}, J=9.0$, $2.5 \mathrm{~Hz}, 4 \mathrm{H}), 7.04$ (dt, $J=9.0,2.5 \mathrm{~Hz}, 4 \mathrm{H}), 3.90$ (s, 6H) ppm; ${ }^{13} \mathrm{CNMR}\left(126 \mathrm{MHz}, \mathrm{CDCl}_{3}\right) \delta=186.9,161.7,159.4,130.2$, 120.5, 120.2, 114.3, 55.5 ppm; IR (KBr) $v=1682$ (s), 1668 (vs) $\mathrm{cm}^{-1}$; MS $m / z(\%)=336\left(\mathrm{M}^{+}, 100\right), 308(24), 293$ (29), 167 (7), 149 (18), 135 (40), 77 (6). HRMS calcd for $\mathrm{C}_{20} \mathrm{H}_{16} \mathrm{O}_{5}\left(\mathrm{M}^{+}\right)$ 336.0998 , found 336.1002 .

\subsection{1,3-diarylisobenzofuran-5,6-dicarbaldoximide 6-8}

To a solution of the 2,5-diaryl-furan-3,4-dicarbaldehyde $(0.5 \mathrm{mmol})$ and $N$-substituted maleimide $(0.55 \mathrm{mmol})$ in $2 \mathrm{ml}$ of dry dioxane was added trioctylphosphine $(0.60 \mathrm{mmol})$. The mixture was refluxed on a preheated oil bath under nitrogen atmosphere for $0.5-2 \mathrm{~h}$, and was cooled to ice-bath temperature. The crystals formed were collected by suction filtration and washed well with cold ether to give the product. If necessary, the filtrate was concentrated under vacuum and the residue was purified by silica gel chromatography with a solvent system of chloroform/ethyl acetate (or 
chloroform/ethanol). The yields are shown in Table 1.

6a: Orange needles, m.p. $285-288^{\circ} \mathrm{C}$. ${ }^{1} \mathrm{HNMR}(500 \mathrm{MHz}$, $\left.\mathrm{CDCl}_{3}\right) \delta=8.51(\mathrm{~s}, 2 \mathrm{H}), 8.00(\mathrm{~d}, J=8.6 \mathrm{~Hz}, 4 \mathrm{H}), 7.56(\mathrm{~m}, 6 \mathrm{H})$ 7.50 (dm, $J=7.4 \mathrm{~Hz}, 2 \mathrm{H}), 7.44$ (tm, $J=7.4 \mathrm{~Hz}, 3 \mathrm{H}) \mathrm{ppm}$; ${ }^{13} \mathrm{CNMR}\left(126 \mathrm{MHz}, \mathrm{CDCl}_{3}\right) \delta=159.3,141.9,124.9,122.9$, $122.3,122.1,121.9,121.3,119.6,119.4,118.7,114.6,113.0$ ppm; IR (KBr) $v=1771(\mathrm{~m}), 1703$ (vs) cm ${ }^{-1}$; UV-vis $\left(\mathrm{CH}_{3} \mathrm{CN}\right)$ $\lambda_{\max }=240 \mathrm{sh}(\log \varepsilon=4.23), 262 \mathrm{sh}$ (4.22), 293 (4.29), 314sh (4.17), 331sh (3.91), 356sh (3.39), 369sh (3.58), 388sh (3.79) 419 (3.91), 441sh (3.89) nm; MS $m / z(\%)=415\left(\mathrm{M}^{+}, 100\right)$, 268 (17), 138 (13). HRMS calcd for $\mathrm{C}_{28} \mathrm{H}_{17} \mathrm{NO}_{3}\left(\mathrm{M}^{+}\right)$ 415.1208, found 415.1212.

6b: Orange solids, m.p. $275-278^{\circ} \mathrm{C} .{ }^{1} \mathrm{HNMR}(500 \mathrm{MHz}$, $\left.\mathrm{CDCl}_{3}\right) \delta=8.40(\mathrm{~s}, 2 \mathrm{H}), 7.97(\mathrm{dd}, J=7.9,1.2 \mathrm{~Hz}, 4 \mathrm{H}), 7.55$ (tm, $J=7.9 \mathrm{~Hz}, 4 \mathrm{H}), 7.43$ (tt, $J=7.9,1.2 \mathrm{~Hz}, 2 \mathrm{H}), 3.24(\mathrm{~s}, 3 \mathrm{H})$ ppm; ${ }^{13} \mathrm{CNMR}\left(126 \mathrm{MHz}, \mathrm{CDCl}_{3}\right) \delta=167.3,148.6,129.9$, 129.2, 128.7, 126.8, 125.5, 121.3, 118.9, 24.3 ppm; IR (KBr) $v$ $=1761(\mathrm{~s}), 1712(\mathrm{vs}) \mathrm{cm}^{-1} ; \mathrm{UV}$-vis $\left(\mathrm{CHCl}_{3}\right) \lambda_{\max }=245(\log \varepsilon=$ 4.20), 251sh (4.20), 271sh (4.24), 283 (4.32), 310sh (4.06), 329sh (3.90), 420 (3.99), 442sh (3.99) nm; UV-vis $\left(\mathrm{CH}_{3} \mathrm{CN}\right)$ $\lambda_{\max }=223(\log \varepsilon=4.32), 241 \mathrm{sh}(4.22), 251 \mathrm{sh}$ (4.22), 263sh (4.25), 280 (4.37), 315sh (4.05), 329sh (3.91), 415 (4.01) 436 (4.01) nm; MS $m / z(\%)=353\left(\mathrm{M}^{+}, 100\right), 268(6), 239$ (22), 177 (6), 134 (8), 77 (6). HRMS calcd for $\mathrm{C}_{23} \mathrm{H}_{15} \mathrm{NO}_{3}\left(\mathrm{M}^{+}\right)$ 353.1052 , found 353.1057 .

6c: Orange solids, m.p. $253-255^{\circ} \mathrm{C} .{ }^{1} \mathrm{HNMR}(500 \mathrm{MHz}$, $\left.\mathrm{CDCl}_{3}\right) \delta=8.37(\mathrm{~s}, 2 \mathrm{H}), 7.96(\mathrm{dm}, J=7.9 \mathrm{~Hz}, 4 \mathrm{H}), 7.54(\mathrm{tm}, J$ $=7.9 \mathrm{~Hz}, 4 \mathrm{H}), 7.42(\mathrm{tt}, J=7.9 \mathrm{~Hz}, 1.4 \mathrm{~Hz}, 2 \mathrm{H}), 3.80(\mathrm{q}, J=7.2$ $\mathrm{Hz}, 2 \mathrm{H}), 1.32$ (t, $J=7.2 \mathrm{~Hz}, 3 \mathrm{H}) \mathrm{ppm} ;{ }^{13} \mathrm{CNMR}(126 \mathrm{MHz}$, $\left.\mathrm{CDCl}_{3}\right) \delta=167.1,148.6,130.0,129.2,128.7,126.9,125.6$, 121.4, 118.9, 33.2, 13.8 ppm; IR (KBr) $v=1750$ (s), $1712(\mathrm{vs})$ $\mathrm{cm}^{-1} ; \mathrm{MS} m / z(\%)=367\left(\mathrm{M}^{+}, 100\right), 268$ (6), 239 (17), 176 (7), 134 (11), 77 (6). HRMS calcd for $\mathrm{C}_{24} \mathrm{H}_{17} \mathrm{NO}_{3}\left(\mathrm{M}^{+}\right) 367.1208$, found 367.1205 .

6d: Orange solid, m.p. $278-281^{\circ} \mathrm{C}$. ${ }^{1} \mathrm{HNMR}(500 \mathrm{MHz}$, $\left.\mathrm{CDCl}_{3}\right) \delta=8.49(\mathrm{~s}, 2 \mathrm{H}), 8.00(\mathrm{dm}, J=7.5 \mathrm{~Hz}, 4 \mathrm{H}), 7.56(\mathrm{tm}, J$ $=7.5 \mathrm{~Hz}, 4 \mathrm{H}), 7.43(\mathrm{tt}, J=7.5,1.4 \mathrm{~Hz}, 2 \mathrm{H}), 7.40(\mathrm{dm}, J=9.2$ $\mathrm{Hz}, 2 \mathrm{H}), 7.05(\mathrm{dm}, J=9.2 \mathrm{~Hz}, 2 \mathrm{H}), 3.87$ (s, 3H) ppm; ${ }^{13} \mathrm{CNMR}\left(126 \mathrm{MHz}, \mathrm{CDCl}_{3}\right) \delta=166.5,159.3,148.8,130.0$, $129.3,128.8,127.9,126.5,125.7,124.5,121.6,119.8,114.5$, $55.5 \mathrm{ppm}$; IR (KBr) $v=1766(\mathrm{~s}), 1706(\mathrm{vs}) \mathrm{cm}^{-1} ; \mathrm{MS} m / z(\%)$ $=445\left(\mathrm{M}^{+}, 100\right), 268(15), 239$ (10), 223 (8). HRMS calcd for $\mathrm{C}_{29} \mathrm{H}_{19} \mathrm{NO}_{3}\left(\mathrm{M}^{+}\right) 445.1314$, found 445.1313 .

7a: Reddish orange powder, m.p. $294-296^{\circ} \mathrm{C} .{ }^{1} \mathrm{HNMR}(500$ $\left.\mathrm{MHz}, \mathrm{CDCl}_{3}\right) \delta=8.47$ (s, 2H), $7.88(\mathrm{~d}, J=8.1 \mathrm{~Hz}, 4 \mathrm{H}), 7.54$ $(\mathrm{tm}, J=7.6 \mathrm{~Hz}, 2 \mathrm{H}), 7.49(\mathrm{dm}, J=7.6 \mathrm{~Hz}, 2 \mathrm{H}), 7.43(\mathrm{tt}, J=$ $7.6,1.7 \mathrm{~Hz}, 1 \mathrm{H}), 7.36(\mathrm{~d}, J=8.1 \mathrm{~Hz}, 4 \mathrm{H}), 2.45$ (s, 6H) ppm; ${ }^{13} \mathrm{CNMR}\left(126 \mathrm{MHz}, \mathrm{CDCl}_{3}\right) \delta=166.3,148.8,139.0,132.0$, $130.0,129.1,128.2,127.3,126.6,126.0,125.6,121.1,120.1$, 21.5 ppm; IR (KBr) $v=1762(\mathrm{~m}), 1711$ (vs) $\mathrm{cm}^{-1}$; UV-vis $\left(\mathrm{CH}_{3} \mathrm{CN}\right) \lambda_{\max }=242 \mathrm{sh}(\log \varepsilon=4.34), 265 \mathrm{sh}$ (4.29), 281 (4.31), 301 (4.36), 320sh (4.24), 339 (3.90), 356 (3.39), 369sh (3.57), 388sh (3.81), 427 (3.98), 460sh (3.95) nm; MS $m / z(\%)=443$ $\left(\mathrm{M}^{+}, 100\right), 296$ (11). HRMS calcd for $\mathrm{C}_{30} \mathrm{H}_{21} \mathrm{NO}_{3}\left(\mathrm{M}^{+}\right)$ 443.1521, found 443.1519.

7b: Reddish orange solids, m.p. $>300^{\circ} \mathrm{C}$. ${ }^{1} \mathrm{HNMR}(500$ $\left.\mathrm{MHz}, \mathrm{CDCl}_{3}\right) \delta=8.32$ (s, 2H), $7.83(\mathrm{~d}, J=7.9 \mathrm{~Hz}, 4 \mathrm{H}), 7.33$ (d, $J=7.9 \mathrm{~Hz}, 4 \mathrm{H}), 3.21(\mathrm{~s}, 3 \mathrm{H}), 2.44(\mathrm{~s}, 6 \mathrm{H}) \mathrm{ppm} ;{ }^{13} \mathrm{CNMR}$ $\left(126 \mathrm{MHz}, \mathrm{CDCl}_{3}\right) \delta=167.4,148.6,138.9,129.9,127.3$, $126.4,125.5,120.9,119.2,24.3,21.5 \mathrm{ppm}$; IR (KBr) $v=1755$ (vs), 1716 (vs), 1701 (vs) $\mathrm{cm}^{-1}$; UV-vis $\left(\mathrm{CH}_{3} \mathrm{CN}\right) \lambda_{\max }=223$ ( $\log \varepsilon=4.50), 240 \mathrm{sh}$ (4.31), 267sh (4.26), 281 (4.32), 292sh (4.28), 319sh (4.08), 338sh (3.82), 369sh (3.62), 388 (3.82), 420 (3.96), 447sh (3.96) nm; MS $m / z(\%)=381\left(\mathrm{M}^{+}, 100\right)$, 253 (5), 191 (8). HRMS calcd for $\mathrm{C}_{25} \mathrm{H}_{19} \mathrm{NO}_{3}\left(\mathrm{M}^{+}\right)$381.1365, found 381.1369 .

7c: Orange solids, m.p. $248-249^{\circ} \mathrm{C} .{ }^{1} \mathrm{HNMR}(500 \mathrm{MHz}$, $\left.\mathrm{CDCl}_{3}\right) \delta=8.34(\mathrm{~s}, 2 \mathrm{H}), 7.84(\mathrm{~d}, J=8.1 \mathrm{~Hz}, 4 \mathrm{H}), 7.34(\mathrm{~d}, J=$ $8.1 \mathrm{~Hz}, 4 \mathrm{H}), 3.79$ (q, $J=7.2 \mathrm{~Hz}, 2 \mathrm{H}), 2.44(\mathrm{~s}, 6 \mathrm{H}), 1.31(\mathrm{t}, J=$ $7.2 \mathrm{~Hz}, 3 \mathrm{H}) \mathrm{ppm} ;{ }^{13} \mathrm{CNMR}\left(126 \mathrm{MHz}, \mathrm{CDCl}_{3}\right) \delta=167.2$, $148.6,138.8,129.9,127.4,126.6,125.5,121.0,119.1,33.2$, 21.5, 13.8 ppm; IR (KBr) $v=1749$ (s), 1701 (vs) $\mathrm{cm}^{-1}$; MS $m / z(\%)=395\left(\mathrm{M}^{+}, 100\right)$. HRMS Calcd for $\mathrm{C}_{26} \mathrm{H}_{21} \mathrm{NO}_{3}\left(\mathrm{M}^{+}\right)$ 395.1521 , found 395.1519 .

$7 \mathrm{~d}$ : Reddish orange solids, m.p. $271-273^{\circ} \mathrm{C} .{ }^{1} \mathrm{HNMR}(500$ $\left.\mathrm{MHz}, \mathrm{CDCl}_{3}\right) \delta=8.46(\mathrm{~s}, 2 \mathrm{H}), 7.89(\mathrm{~d}, J=8.2 \mathrm{~Hz}, 4 \mathrm{H}), 7.39$ $(\mathrm{dt}, J=8.9,2.8 \mathrm{~Hz}, 2 \mathrm{H}), 7.35(\mathrm{~d}, J=8.2 \mathrm{~Hz}, 4 \mathrm{H}), 7.05(\mathrm{dt}, J=$ $8.9,2.8 \mathrm{~Hz}, 2 \mathrm{H}), 3.89$ (s, 3H), 2.45 (s, 6H) ppm; ${ }^{13} \mathrm{CNMR}(126$ $\left.\mathrm{MHz}, \mathrm{CDCl}_{3}\right) \delta=166.6,159.3,148.7,138.9,130.0,127.9$, $127.3,126.1,125.5,124.6,121.0,119.9,114.4,55.5,21.5$ ppm; IR (KBr) $v=1759$ (s), $1712(\mathrm{vs}) \mathrm{cm}^{-1}$; MS $m / z(\%)=$ $473\left(\mathrm{M}^{+}, 100\right), 296$ (10), 237 (9). HRMS calcd for $\mathrm{C}_{31} \mathrm{H}_{23} \mathrm{NO}_{4}$ $\left(\mathrm{M}^{+}\right)$473.1627, found 473.1632 .

7e: Orange solids, m.p. $229-232^{\circ} \mathrm{C} .{ }^{1} \mathrm{HNMR}(500 \mathrm{MHz}$, $\left.\mathrm{CDCl}_{3}\right) \delta=8.30(\mathrm{~s}, 2 \mathrm{H}), 7.84(\mathrm{~d}, J=8.1 \mathrm{~Hz}, 4 \mathrm{H}), 7.33(\mathrm{~d}, J=$ $8.1 \mathrm{~Hz}, 4 \mathrm{H}), 4.19(\mathrm{tt}, J=3.6 \mathrm{~Hz}, 12.3 \mathrm{~Hz}, 1 \mathrm{H}), 2.44(\mathrm{~s}, 6 \mathrm{H})$ 2.28 (qd, $J=12.3,3.6 \mathrm{~Hz}, 2 \mathrm{H}), 1.89$ (d, $J=12.3 \mathrm{~Hz}, 2 \mathrm{H})$, $1.70-1.77(\mathrm{~m}, J=12.3,3.6 \mathrm{~Hz}, 3 \mathrm{H}), 1.27-1.44(\mathrm{~m}, J=12.3$, $3.6 \mathrm{~Hz}, 3 \mathrm{H}) \mathrm{ppm} ;{ }^{13} \mathrm{CNMR}\left(126 \mathrm{MHz}, \mathrm{CDCl}_{3}\right) \delta=167.6$, $148.6,138.9,130.1,127.6,126.7,125.6,121.2,119.0,51.4$, 29.7, 26.2, 25.3, 21.6 ppm; IR (KBr) $v=1755$ (s), 1701 (vs) $\mathrm{cm}^{-1}$; MS $\mathrm{m} / z(\%)=449\left(\mathrm{M}^{+}, 100\right), 367$ (28). HRMS Calcd for $\mathrm{C}_{30} \mathrm{H}_{27} \mathrm{NO}_{3}\left(\mathrm{M}^{+}\right)$449.1991, found 449.1987.

8a: Reddish orange solids, m.p. $263-264^{\circ} \mathrm{C} .{ }^{1} \mathrm{HNMR}(500$ $\left.\mathrm{MHz}, \mathrm{CDCl}_{3}\right) \delta=8.41(\mathrm{~s}, 2 \mathrm{H}), 7.90(\mathrm{dt}, J=8.9,2.4 \mathrm{~Hz}, 4 \mathrm{H})$, $7.53(\mathrm{t}, J=7.4 \mathrm{~Hz}, 2 \mathrm{H}), 7.48(\mathrm{~d}, J=7.4 \mathrm{~Hz}, 2 \mathrm{H}), 7.42$ (t, $J=$ $7.4 \mathrm{~Hz}, 1 \mathrm{H}), 7.07$ (dt, $J=8.9,2.4 \mathrm{~Hz}, 4 \mathrm{H}), 3.90$ (s, 6H) ppm; ${ }^{13} \mathrm{CNMR}\left(126 \mathrm{MHz}, \mathrm{CDCl}_{3}\right) \delta=166.4,160.0,148.4,132.0$, 129.1, 128.2, 127.1, 126.6, 125.8, 123.0, 120.4, 120.2, 114.8, 55.5 ppm; IR (KBr) $v=1760(\mathrm{~s}), 1707$ (vs) $\mathrm{cm}^{-1}$; UV-vis $\left(\mathrm{CH}_{3} \mathrm{CN}\right) \lambda_{\max }=233(\log \varepsilon=4.20), 270$ (4.29), 279 (4.36), 308 (4.49), 434 (4.11), 481sh (4.06) nm; MS $m / z(\%)=475\left(\mathrm{M}^{+}\right.$, 100), 460 (29), 368 (7), 238 (12), 164 (7), 111 (6), 97 (10), 83 (10), 71 (10). HRMS calcd for $\mathrm{C}_{30} \mathrm{H}_{21} \mathrm{NO}_{5}\left(\mathrm{M}^{+}\right)$475.1420, found 475.1420 .

8b: Reddish orange solids, m.p. $277-278^{\circ} \mathrm{C}$. ${ }^{1} \mathrm{HNMR}(500$ $\left.\mathrm{MHz}, \mathrm{CDCl}_{3}\right) \delta=8.29(\mathrm{~s}, 2 \mathrm{H}), 7.87(\mathrm{dt}, J=8.9,2.5 \mathrm{~Hz}, 4 \mathrm{H})$, 7.07 (dt, $J=8.9,2.5 \mathrm{~Hz}, 4 \mathrm{H}), 3.90$ (s. $6 \mathrm{H}), 3.22$ (s, 3H) ppm; ${ }^{13} \mathrm{CNMR}\left(126 \mathrm{MHz}, \mathrm{CDCl}_{3}\right) \delta=167.5,159.9,148.3,127.0$, 126.2, 123.0, 120.2, 119.2, 114.8, 55.5, 24.3 ppm; IR (KBr) $v$ $=1756(\mathrm{vs}), 1714(\mathrm{~s}) \mathrm{cm}^{-1} ; \mathrm{MS} m / z(\%)=413\left(\mathrm{M}^{+}, 100\right), 398$ (40), 207 (16). HRMS calcd for $\mathrm{C}_{25} \mathrm{H}_{19} \mathrm{NO}_{5}\left(\mathrm{M}^{+}\right)$413.1263, found 413.1263 .

8d: Carmine solids, m.p. $230-231^{\circ} \mathrm{C} .{ }^{1} \mathrm{HNMR}(500 \mathrm{MHz}$, 
$\left.\mathrm{CDCl}_{3}\right) \delta=8.38(\mathrm{~s}, 2 \mathrm{H}), 7.88(\mathrm{dt}, J=9.0,2.4 \mathrm{~Hz}, 4 \mathrm{H}), 7.38(\mathrm{dt}$, $J=8.9,2.7 \mathrm{~Hz}, 2 \mathrm{H}), 7.06(\mathrm{dtt}, J=9.0,2.4 \mathrm{~Hz}, 4 \mathrm{H}), 7.04(\mathrm{dt}, J$ $=8.9,2.7 \mathrm{~Hz}, 2 \mathrm{H}), 3.90(\mathrm{~s}, 6 \mathrm{H}), 3.86(\mathrm{~s}, 3 \mathrm{H}) \mathrm{ppm} ;{ }^{13} \mathrm{CNMR}$ $\left(126 \mathrm{MHz}, \mathrm{CDCl}_{3}\right) \delta=166.6,160.0,159.3,148.4,127.9$, 127.0, 125.8, 124.7, 123.0, 120.4, 120.0, 114.8, 114.4, 55.52, $55.47 \mathrm{ppm}$; IR (KBr) $v=1757(\mathrm{~m}), 1712(\mathrm{vs}) \mathrm{cm}^{-1}$; MS m/z $(\%)=505\left(\mathrm{M}^{+}, 100\right), 490$ (24), 253 (11). HRMS calcd for $\mathrm{C}_{31} \mathrm{H}_{23} \mathrm{NO}_{6}\left(\mathrm{M}^{+}\right)$505.1525, found 505.1520.

\subsection{1,4-diarylnaphthalene-2,3:6,7-bis(dicarboximide)s 18-20}

A solution of 1,3-diarylisobenzofuran-5,6-dicarboximide $(1.00 \mathrm{mmol})$ and $N$-substituted maleimide $(1.10 \mathrm{mmol})$ in 10 $\mathrm{mL}$ of toluene or dioxane was refluxed on an oil bath under argon atmosphere until the isobenzofuran was consumed completely $(5-20 \mathrm{~h})$. Then, the solvent was removed and the residue was purified by $\mathrm{SiO}_{2}$ chromatography to give the Diels-Alder adduct $(63-99 \%$ yield). This adduct was dissolved in a solution of 10 equivalents of trifluoromethanesulfonic acid in $5 \mathrm{~mL}$ of DCE. After being heated at $65^{\circ} \mathrm{C}$ for $2 \mathrm{~h}$, the resulted reaction mixture was poured into water and was extracted with chloroform $(20 \mathrm{~mL} \mathrm{x}$ 3 ). The combined organic layer was washed with a saturated $\mathrm{NaHCO}_{3}$ solution and brine, and was dired over $\mathrm{Na}_{2} \mathrm{SO}_{4}$. The solvent was removed and the residue was purified by $\mathrm{SiO}_{2}$ chromatography to give the title compound. Two-step yields are shown in Table 3.

18a: Colorless powder, m.p. $>300^{\circ} \mathrm{C} .{ }^{1} \mathrm{HNMR}(500 \mathrm{MHz}$, $\left.\mathrm{CDCl}_{3}\right) \delta=8.30(\mathrm{~s}, 2 \mathrm{H}), 7.61(\mathrm{~m}, 6 \mathrm{H}), 7.42(\mathrm{~m}, 4 \mathrm{H}), 4.04(\mathrm{tt}, J$ $=12.8,3.4 \mathrm{~Hz}, 1 \mathrm{H}), 3.20(\mathrm{~s}, 3 \mathrm{H}), 2.13(\mathrm{qd}, J=12.8,3.4 \mathrm{~Hz}$, 2H), 1.77 (d, $J=12.8 \mathrm{~Hz}, 2 \mathrm{H}), 1.64$ (d, $J=12.8 \mathrm{~Hz}, 2 \mathrm{H}), 1.59$ $(\mathrm{d}, J=12.8 \mathrm{~Hz}, 1 \mathrm{H}), 1.23(\mathrm{qt}, J=12.8,3.4 \mathrm{~Hz}, 2 \mathrm{H}), 1.13$ (qt, $J$ $=12.8,3.4 \mathrm{~Hz}, 1 \mathrm{H}) \mathrm{ppm} ;{ }^{13} \mathrm{CNMR}\left(126 \mathrm{MHz}, \mathrm{CDCl}_{3}\right) \delta=$ $167.5,166.4,140.8,138.7,133.8,130.1,129.8,129.3,128.7$, 126.6, 124.6, 51.6, 29.6, 26.1, 25.1, $24.6 \mathrm{ppm}$; IR (KBr) $v=$ 1766 (s), 1718 (vs), 1707 (vs) $\mathrm{cm}^{-1}$; UV $\left(\mathrm{CHCl}_{3}\right) \lambda_{\max }=251$ ( $\log \varepsilon=4.62), 268$ (4.77), 315 (4.07), 369 (3.69), 387 (3.88) $\mathrm{nm} ; \mathrm{MS} m / z(\%)=514\left(\mathrm{M}^{+}, 66\right), 433(100), 415(6), 301$ (7), 276 (11). HRMS calcd for $\mathrm{C}_{33} \mathrm{H}_{26} \mathrm{~N}_{2} \mathrm{O}_{4}\left(\mathrm{M}^{+}\right)$514.1893, found 514.1887.

18b: Creamy white powder, m.p. $>300^{\circ} \mathrm{C}$ (sintered above $\left.300^{\circ} \mathrm{C}\right) .{ }^{1} \mathrm{HNMR}\left(500 \mathrm{MHz}, \mathrm{CDCl}_{3}\right) \delta=8.36(\mathrm{~s}, 2 \mathrm{H}), 7.60(\mathrm{~m}$, $6 \mathrm{H}), 7.46(\mathrm{~m}, 4 \mathrm{H}), 7.38(\mathrm{tm}, J=7.5 \mathrm{~Hz}, 2 \mathrm{H}), 7.35(\mathrm{dt}, J=6.9$, $2.0 \mathrm{~Hz}, 2 \mathrm{H}), 7.31(\mathrm{tt}, J=6.9,2.0 \mathrm{~Hz}, 1 \mathrm{H}), 3.78(\mathrm{q}, J=7.2 \mathrm{~Hz}$, $2 \mathrm{H}), 1.27(\mathrm{t}, J=7.2 \mathrm{~Hz}, 3 \mathrm{H}) \mathrm{ppm} ;{ }^{13} \mathrm{CNMR}\left(126 \mathrm{MHz}, \mathrm{CDCl}_{3}\right)$ $\delta=167.0,165.2,141.6,138.7,133.4,131.3,130.2,129.7$, $129.2,128.7,128.6,128.1,126.6,126.1,124.5,33.4,13.8$ ppm; IR (KBr) $v=1768$ (s), 1727 (vs), 1718 (vs), 1711 (s) $\mathrm{cm}^{-}$ ${ }^{1}$; UV $\left(\mathrm{CHCl}_{3}\right) \lambda_{\max }=248 \mathrm{sh}(\log \varepsilon=4.59), 263$ (4.76), 274sh (4.69), 313sh (4.23), 370 (3.71), $388(3.86) \mathrm{nm} ; \mathrm{MS} \mathrm{m} / z(\%)=$ $522\left(\mathrm{M}^{+}, 100\right), 477$ (15), 423 (5), 378 (6), 301 (4), 276 (5). HRMS calcd for $\mathrm{C}_{34} \mathrm{H}_{22} \mathrm{~N}_{2} \mathrm{O}_{4}\left(\mathrm{M}^{+}\right)$522.1580, found 522.1586.

18c: Yellow powder, m.p. $>300^{\circ} \mathrm{C} .{ }^{1} \mathrm{HNMR}(500 \mathrm{MHz}$, $\left.\mathrm{CDCl}_{3}\right) \delta=8.48(\mathrm{~s}, 2 \mathrm{H}), 7.61(\mathrm{~m}, 6 \mathrm{H}), 7.51(\mathrm{~m}, 6 \mathrm{H}), 7.40(\mathrm{~m}$, $7 \mathrm{H}), 7.32$ (tt, $J=6.9,1.9 \mathrm{~Hz}, 1 \mathrm{H}) \mathrm{ppm} ;{ }^{13} \mathrm{CNMR}(126 \mathrm{MHz}$, $\left.\mathrm{CDCl}_{3}\right) \delta=166.1,165.2,141.7,139.0,133.3,131.4,131.3$, $129.70,129.67,129.3,129.2,128.8,128.7,128.5,128.2$,
126.6, 126.5, 126.3, 125.3 ppm; IR (KBr) $v=1772$ (s), 1744 (s), 1725 (vs) cm ${ }^{-1}$; UV $\left(\mathrm{CHCl}_{3}\right) \lambda_{\max }=259$ (log $\left.\varepsilon=4.68\right), 288$ (4.52), 370 (3.70), $389(3.82) \mathrm{nm}$. MS $m / z(\%)=570\left(\mathrm{M}^{+}\right.$, 100), 525 (14), 378 (9). HRMS calcd for $\mathrm{C}_{38} \mathrm{H}_{22} \mathrm{~N}_{2} \mathrm{O}_{4}\left(\mathrm{M}^{+}\right)$ 570.1580 , found 570.1583 .

18d: Yellow microcrystals, m.p. $>300^{\circ} \mathrm{C}$. ${ }^{1} \mathrm{HNMR}(500$ $\left.\mathrm{MHz}, \mathrm{CDCl}_{3}\right) \delta=8.41(\mathrm{~s}, 2 \mathrm{H}), 7.62(\mathrm{~m}, 6 \mathrm{H}), 7.45(\mathrm{~m}, 4 \mathrm{H})$, $7.30(\mathrm{~d}, J=9.1 \mathrm{~Hz}, 2 \mathrm{H}), 7.02(\mathrm{~d}, J=9.1 \mathrm{~Hz}, 2 \mathrm{H}), 3.84(\mathrm{~s}, 3 \mathrm{H})$, $3.66(\mathrm{q}, J=7.2 \mathrm{~Hz}, 2 \mathrm{H}), 1.20(\mathrm{t}, J=7.2 \mathrm{~Hz}, 3 \mathrm{H}) \mathrm{ppm}$; ${ }^{13} \mathrm{CNMR}\left(126 \mathrm{MHz}, \mathrm{CDCl}_{3}\right) \delta=166.4,166.1,159.4,140.9$, $138.7,133.5,130.0,129.6,129.2,128.6,127.8,126.8,125.1$, 124.0, 114.5, 55.5, 33.3, $13.6 \mathrm{ppm}$; IR (KBr) $v=1766$ (s), 1732 (s), 1717 (vs), 1706 (s) $\mathrm{cm}^{-1}$; UV $\left(\mathrm{CHCl}_{3}\right) \lambda_{\max }=263$ ( $\log \varepsilon=4.75), 309$ (4.25), 369 (3.72), 388 (3.79) nm; MS m/z $(\%)=552\left(\mathrm{M}^{+}, 100\right), 537$ (7), $276(6), 269$ (8), HRMS calcd for $\mathrm{C}_{35} \mathrm{H}_{24} \mathrm{~N}_{2} \mathrm{O}_{5}\left(\mathrm{M}^{+}\right)$552.1685, found 552.1687.

19a: Creamy white powder, m.p. $>300^{\circ} \mathrm{C}$. ${ }^{1} \mathrm{HNMR}(500$ $\left.\mathrm{MHz}, \mathrm{CDCl}_{3}\right) \delta=8.32(\mathrm{~s}, 2 \mathrm{H}), 7.41(\mathrm{~d}, J=7.9 \mathrm{~Hz}, 4 \mathrm{H}), 7.30$ $(\mathrm{d}, J=7.9 \mathrm{~Hz}, 4 \mathrm{H}), 4.05(\mathrm{tt}, J=12.5,3.5 \mathrm{~Hz}, 1 \mathrm{H}), 3.20(\mathrm{~s}, 3 \mathrm{H})$, $2.53(\mathrm{~s}, 6 \mathrm{H}), 2.14(\mathrm{qd}, J=12.5,3.5 \mathrm{~Hz}, 2 \mathrm{H}), 1.77(\mathrm{~d}, J=12.5$ $\mathrm{Hz}, 2 \mathrm{H}), 1.64$ (d, $J=12.5 \mathrm{~Hz}, 2 \mathrm{H}), 1.59$ (d, $J=12.5 \mathrm{~Hz}, 1 \mathrm{H})$, 1.23 (qt, $J=12.5,3.5 \mathrm{~Hz}, 2 \mathrm{H}), 1.12$ (qt, $J=12.5,3.5 \mathrm{~Hz}, 1 \mathrm{H})$ ppm; ${ }^{13} \mathrm{CNMR}\left(126 \mathrm{MHz}, \mathrm{CDCl}_{3}\right) \delta=167.6,166.6,141.0$, $139.1,139.0,130.7,129.9,129.7,129.5,126.6,124.7,51.5$, 29.6, 26.1, 25.2, 24.6, 21.7 ppm; IR (KBr) $v=1761$ (s), 1716 (vs), 1704 (vs) cm $\mathrm{cm}^{-1} \mathrm{UV}\left(\mathrm{CHCl}_{3}\right) \lambda_{\max }=252(\log \varepsilon=4.60)$, 268 (4.78), 318sh (3.95), 339sh (3.83), 372 (3.66), 389 (3.81) $\mathrm{nm} ; \mathrm{MS} m / z(\%)=542\left(\mathrm{M}^{+}, 95\right), 461(100), 445$ (11). HRMS Calcd for $\mathrm{C}_{35} \mathrm{H}_{30} \mathrm{~N}_{2} \mathrm{O}_{4}\left(\mathrm{M}^{+}\right)$542.2206, found 542.2202.

19b: Yellow powder, m.p. $>300^{\circ} \mathrm{C} .{ }^{1} \mathrm{HNMR}(500 \mathrm{MHz}$, $\left.\mathrm{CDCl}_{3}\right) \delta=8.38(\mathrm{~s}, 2 \mathrm{H}) 7.29-7.40(\mathrm{~m}, 13 \mathrm{H}), 3.78$ (q, $J=7.2$ $\mathrm{Hz}, 2 \mathrm{H}), 2.51(\mathrm{~s}, 6 \mathrm{H}), 1.27(\mathrm{t}, J=7.2 \mathrm{~Hz}, 3 \mathrm{H}) \mathrm{ppm} ;{ }^{13} \mathrm{CNMR}$ $\left(126 \mathrm{MHz}, \mathrm{CDCl}_{3}\right) \delta=167.2,165.5,141.9,139.2,139.1$, $131.5,130.6,130.3,129.8,129.5,128.8,128.2,126.7,126.2$, 124.8, 33.6, 21.7, 14.0 ppm; IR (KBr) $v=1767$ (s), 1731 (vs), 1721 (vs) 1717 (s, sh), $1711(\mathrm{~s}, \mathrm{sh}) \mathrm{cm}^{-1}$; MS $m / z(\%)=550$ $\left(\mathrm{M}^{+}, 100\right), 535$ (28), 505 (11), 491 (6). HRMS Calcd for $\mathrm{C}_{36} \mathrm{H}_{26} \mathrm{~N}_{2} \mathrm{O}_{4}\left(\mathrm{M}^{+}\right)$550.1893, found 550.1893.

19c: Yellow powder, m.p. $>300^{\circ} \mathrm{C}$. ${ }^{1} \mathrm{HNMR}(500 \mathrm{MHz}$, $\left.\mathrm{CDCl}_{3}\right) \delta=8.51(\mathrm{~s}, 2 \mathrm{H}), 7.52(\mathrm{tm}, J=7.2 \mathrm{~Hz}, 2 \mathrm{H}), 7.42(\mathrm{~m}$, $7 \mathrm{H}), 7.38(\mathrm{~m}, 8 \mathrm{H}), 7.31(\mathrm{~m}, 1 \mathrm{H}), 2.52(\mathrm{~s}, 6 \mathrm{H}) \mathrm{ppm} ;{ }^{13} \mathrm{CNMR}$ $\left(126 \mathrm{MHz}, \mathrm{CDCl}_{3}\right) \delta=166.4,165.5,142.0,139.4,139.3$, $131.7,131.5,130.5,129.8,129.7,129.6,129.4,128.9,128.6$, $128.3,126.72,126.66,126.4,125.6,21.7 \mathrm{ppm}$; IR (KBr) $v=$ 1769 (s), 1748 (s), 1731 (vs), 1721 (vs) cm ${ }^{-1}$; UV ( $\left.\mathrm{CHCl}_{3}\right)$ $\lambda_{\max }=261(\log \varepsilon=4.77), 283$ (4.64), 325sh (4.22), 342sh (4.05), 364sh (3.78), $392(3.82) \mathrm{nm} ; \mathrm{MS} m / z(\%)=598\left(\mathrm{M}^{+}\right.$, 100), 583 (19), 553 (9), 406 (5), 299 (7). HRMS Calcd for $\mathrm{C}_{40} \mathrm{H}_{26} \mathrm{~N}_{2} \mathrm{O}_{4}\left(\mathrm{M}^{+}\right) 598.1893$ found 598.1901.

19d: Yellow solids, m.p. $>300^{\circ} \mathrm{C}$. ${ }^{1} \mathrm{HNMR}(500 \mathrm{MHz}$, $\left.\mathrm{CDCl}_{3}\right) \delta=8.44(\mathrm{~s}, 2 \mathrm{H}), 742(\mathrm{~d}, J=8.2 \mathrm{~Hz}, 4 \mathrm{H}), 7.33(\mathrm{~d}, J=$ $8.2 \mathrm{~Hz}, 4 \mathrm{H}), 7.31(\mathrm{dm}, J=9.1 \mathrm{~Hz}, 2 \mathrm{H}), 7.02(\mathrm{dm}, J=9.1 \mathrm{~Hz}$, $2 \mathrm{H}), 3.84(\mathrm{~s}, 3 \mathrm{H}), 3.65(\mathrm{q}, J=7.2 \mathrm{~Hz}, 2 \mathrm{H}), 2.53(\mathrm{~s}, 6 \mathrm{H}), 1.20(\mathrm{t}$, $J=7.2 \mathrm{~Hz}, 3 \mathrm{H}) \mathrm{ppm} ;{ }^{13} \mathrm{CNMR}\left(126 \mathrm{MHz}, \mathrm{CDCl}_{3}\right) \delta=166.7$, $166.4,159.6,141.1,139.2,139.1,130.6,129.8,129.6,129.5$, 128.0, 126.9, 125.4, 124.2, 114.7, 55.7, 33.4, 21.7, 13.7 ppm; IR (KBr) $v=1766$ (s), 1730 (s), 1716 (vs) 1707 (s) $\mathrm{cm}^{-1}$; UV 
$\left(\mathrm{CHCl}_{3}\right) \lambda_{\max }=264$ (log $\left.\varepsilon=4.89\right), 314 \mathrm{sh}$ (4.28), 370sh (3.78), $389(3.83) \mathrm{nm} ; \mathrm{UV}\left(\mathrm{CH}_{3} \mathrm{CN}\right) \lambda_{\max }=228(\log \varepsilon=4.55), 236$ (4.59), 261 (4.84), 302sh (4.31), 367 (3.71), 386 (3.78) nm; MS $m / z(\%)=580\left(\mathrm{M}^{+}, 100\right), 565(10), 283$ (5). HRMS Calcd for $\mathrm{C}_{37} \mathrm{H}_{28} \mathrm{~N}_{2} \mathrm{O}_{5}\left(\mathrm{M}^{+}\right)$580.1998, found 580.2001.

20a: Bright yellow solids, m.p. $>300^{\circ} \mathrm{C}$. ${ }^{1} \mathrm{HNMR}(500 \mathrm{MHz}$, $\left.\mathrm{CDCl}_{3}\right) \delta=8.34(\mathrm{~s}, 2 \mathrm{H}), 7.34(\mathrm{dm}, J=8.8 \mathrm{~Hz}, 4 \mathrm{H}), 7.13(\mathrm{dm}$, $J=8.8 \mathrm{~Hz}, 4 \mathrm{H}), 4.06(\mathrm{tt}, J=12.5,3.3 \mathrm{~Hz}, 1 \mathrm{H}), 3.95(\mathrm{~s}, 6 \mathrm{H})$, $3.21(\mathrm{~s}, 3 \mathrm{H}), 2.15(\mathrm{qd}, J=12.5,3.3 \mathrm{~Hz}, 2 \mathrm{H}), 1.78(\mathrm{~d}, J=12.5$ $\mathrm{Hz}, 2 \mathrm{H}), 1.65$ (d, $J=12.5 \mathrm{~Hz}, 2 \mathrm{H}), 1.60$ (d, $J=12.5 \mathrm{~Hz}, 1 \mathrm{H})$, 1.24 (qt, $J=12.5,3.3 \mathrm{~Hz}, 2 \mathrm{H}), 1.14$ (qt, $J=12.5,3.3 \mathrm{~Hz}, 1 \mathrm{H}$ ) ppm; ${ }^{13} \mathrm{CNMR}\left(126 \mathrm{MHz}, \mathrm{CDCl}_{3}\right) \delta=167.4,166.5,160.1$, $140.5,139.0,131.1,129.8,126.5,125.5,124.6,114.1,55.3$, 51.4, 29.5, 26.0, 25.0, $24.5 \mathrm{ppm}$; IR (KBr) $v=1779 \mathrm{~m}, 1760 \mathrm{~s}$, $1716 \mathrm{~s}, 1702 \mathrm{vs} \mathrm{cm}^{-1}$; UV $\left(\mathrm{CHCl}_{3}\right) \lambda_{\max }=250(\log \varepsilon=4.55)$, 270 (4.76), 300 (3.89), 358 (3.80), 385 (3.69) nm; MS $m / z(\%)$ $=574\left(\mathrm{M}^{+}, 100\right), 492(74)$. HRMS calcd for $\mathrm{C}_{35} \mathrm{H}_{30} \mathrm{~N}_{2} \mathrm{O}_{6}\left(\mathrm{M}^{+}\right)$ 574.2104, found 574.2111.

20b: Bright yellow solids, m.p. $>300^{\circ} \mathrm{C}$. ${ }^{1} \mathrm{HNMR}(500 \mathrm{MHz}$,
$\left.\mathrm{CDCl}_{3}\right) \delta=8.46(\mathrm{~s}, 2 \mathrm{H}), 7.37(\mathrm{dm}, J=8.8 \mathrm{~Hz}, 4 \mathrm{H}), 7.31(\mathrm{dm}$, $J=9.1 \mathrm{~Hz}, 2 \mathrm{H}), 7.14(\mathrm{dm}, J=8.8 \mathrm{~Hz}, 4 \mathrm{H}), 7.02(\mathrm{dm}, J=9.1$ $\mathrm{Hz}, 2 \mathrm{H}), 3.95$ (s, 6H), 3.85 (s, 3H), 3.67 (q, $J=7.2 \mathrm{~Hz}, 2 \mathrm{H})$, $1.21(\mathrm{t}, J=7.2 \mathrm{~Hz}, 3 \mathrm{H}) \mathrm{ppm} ;{ }^{13} \mathrm{CNMR}\left(126 \mathrm{MHz}, \mathrm{CDCl}_{3}\right) \delta=$ $166.6,166.3,160.2,159.4,140.7,139.2,131.2,129.5,127.8$, 126.6, 125.4, 125.2, 124.1, 114.5, 114.1, 55.5, 55.4, 33.3, 13.6 ppm; IR (KBr) $v=1765$ (s), 1730 (s), 1716 (vs), 1707 (s) cm ${ }^{1}$; MS $m / z(\%)=612\left(\mathrm{M}^{+}, 100\right), 306(7)$. HRMS Calcd for $\mathrm{C}_{37} \mathrm{H}_{28} \mathrm{~N}_{2} \mathrm{O}_{7}\left(\mathrm{M}^{+}\right)$612.1897, found 612.1896.

\section{Results and Discussion}

Furandicarbaldehydes 9-11, prepared from the corresponding dimethyl diesters $12-14$ [25] by $\mathrm{LiAlH}_{4}$ reduction and subsequent Swern oxidation, were subjected to the annulation reaction with various $N$-substituted maleimides to produce the title isobenzofurans $6-8$ in a range of 50 to $74 \%$ yields (Scheme 2, Table 1). Compounds 6-8 were obtained as slightly air-sensitive orange to red solids.<smiles>COC(=O)c1c([Al])oc([Al])c1C(=O)OC</smiles>

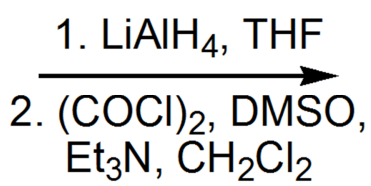

$$
\begin{aligned}
& \text { 12: } \mathrm{Ar}=\mathrm{Ph} \\
& \text { 13: } \mathrm{Ar}=4-\mathrm{MeC}_{6} \mathrm{H}_{4} \\
& \text { 14: } \mathrm{Ar}=4-\mathrm{MeOC}_{6} \mathrm{H}_{4}
\end{aligned}
$$

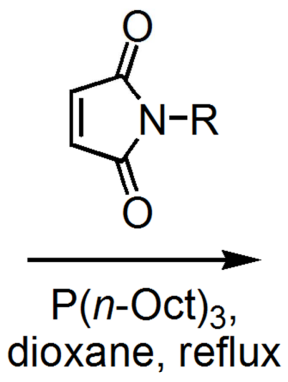<smiles>[R]N1C(=O)c2cc3c([Al])oc([Al])c3cc2C1=O</smiles>

6: $\mathrm{Ar}=\mathrm{Ph}$

7: $\mathrm{Ar}=4-\mathrm{MeC}_{6} \mathrm{H}_{4}$

8: $\mathrm{Ar}=4-\mathrm{MeOC}_{6} \mathrm{H}_{4}$

Scheme 2. Synthesis of 1,3-diarylisobenzofuran-5,6-dicarboximides (6-8).

The UV-vis and emission spectra of $6 \mathrm{a}-8 \mathrm{a}$ are shown in Figure 1. The absorption spectra show mainly two bands around 300 and $450 \mathrm{~nm}$. The latter long wavelength band is very broad, suggesting that it has two excitations. Based on a result of TD-DFT calculations, the band consists of two excitations derived from HOMO $\rightarrow$ LUMO and HOMO $\rightarrow$ LUMO+1.[26] The spectra of 7a and 8a show a clear red shift and also a hyperchromic effect compared with the spectrum of $6 a$.

Table 1. Yield of 1,3-diarylisobenzofuran-5,6-dicarboximides (6-8).

\begin{tabular}{llll}
\hline $\mathbf{A r}$ & $\mathbf{R}$ & product & Yield (\%) \\
\hline $\mathrm{Ph}$ & $\mathrm{Ph}$ & $6 \mathrm{a}$ & 68 \\
$\mathrm{Ph}$ & $\mathrm{Me}$ & $6 \mathrm{~b}$ & 67 \\
$\mathrm{Ph}$ & $\mathrm{Et}$ & $6 \mathrm{c}$ & 74 \\
$\mathrm{Ph}$ & $4-\mathrm{MeOC}_{6} \mathrm{H}_{4}$ & $6 \mathrm{~d}$ & 66 \\
$4-\mathrm{MeC}_{6} \mathrm{H}_{4}$ & $\mathrm{Ph}$ & $7 \mathrm{a}$ & 59 \\
$4-\mathrm{MeC}_{6} \mathrm{H}_{4}$ & $\mathrm{Me}$ & $7 \mathrm{~b}$ & 63 \\
$4-\mathrm{MeC}_{6} \mathrm{H}_{4}$ & $\mathrm{Et}$ & $7 \mathrm{c}$ & 73 \\
$4-\mathrm{MeC}_{6} \mathrm{H}_{4}$ & $4-\mathrm{MeOC} \mathrm{H}_{4}$ & $7 \mathrm{~d}$ & 51 \\
$4-\mathrm{MeC}_{6} \mathrm{H}_{4}$ & $\mathrm{c}-h e x$ & $7 \mathrm{e}$ & 51 \\
$4-\mathrm{MeOC}_{6} \mathrm{H}_{4}$ & $\mathrm{Ph}$ & $8 \mathrm{a}$ & 50 \\
$4-\mathrm{MeOC}_{6} \mathrm{H}_{4}$ & $\mathrm{Me}$ & $8 \mathrm{~b}$ & 70 \\
$4-\mathrm{MeOC}_{6} \mathrm{H}_{4}$ & $4-\mathrm{MeOC}_{6} \mathrm{H}_{4}$ & $8 \mathrm{~d}$ & 66 \\
\hline
\end{tabular}

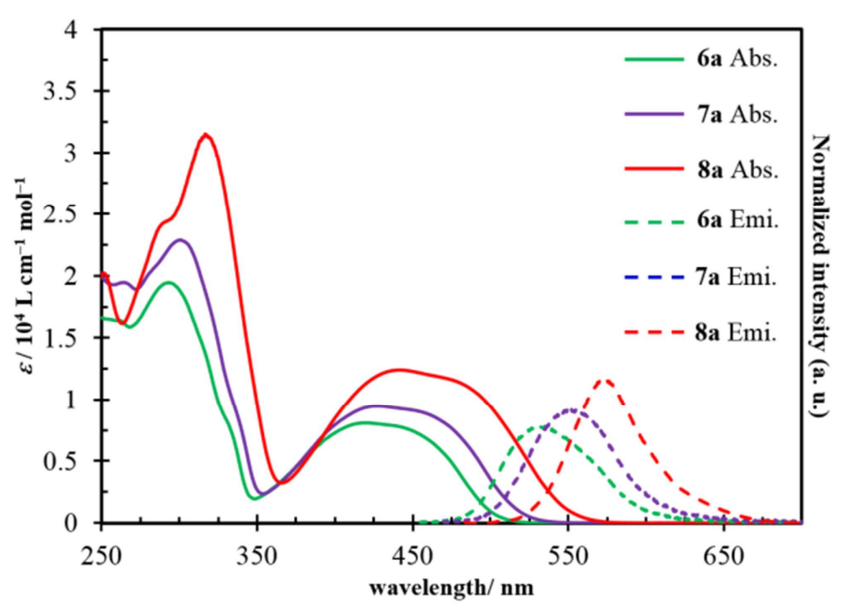

Figure 1. UV-vis absorption (solid line) and emission (broken line) spectra of $6 a-8 a$.

Absorption and emission properties of $6 \mathrm{a}-8 \mathrm{a}$ in acetonitrile are summarized in Table 2. Emission quantum yields were found a range of $1-4 \%$, which are far less than those of 1,3-diphenylisobenzofuran (98\%), its 5,6-diester (72\%) and 
its 5,6-dinitrile (80\%) [27-29].

Table 2. Optical properties of N-phenyl-1,3-diarylisobenzofuran-5,6dicarboximides $(6 a-8 a)$ in acetonitrile.

\begin{tabular}{llllll}
\hline imides & $\lambda_{\text {abs }} / \mathbf{n m}$ & $\log \varepsilon$ & $\lambda_{\text {emi }} /$ nm & Stokes shift $/ \mathbf{n m}$ & $\boldsymbol{\Phi} / \%$ \\
\hline \multirow{2}{*}{ 6a } & 419 & 3.91 & 534 & 115 & 4 \\
& $441 \mathrm{sh}$ & 3.89 & & 93 & 2 \\
7a & 427 & 3.98 & 552 & 125 & \\
& $460 \mathrm{sh}$ & 3.95 & & 92 & \\
8a & 434 & 4.11 & 572 & 138 & 1 \\
\hline
\end{tabular}

Next, compounds 6-8 were employed as an enophile in cycloaddition reaction. The Diels-Alder reaction of 6-8 with another maleimides and subsequent dehydration with the aid of trifluoromethanesulfonic acid in dichloroethane (DCE) produced naphthalene-2,3:6,7-bis(dicarboximide)s. (Scheme 3) Two-step yields of 18-20 via 15-17 are shown in Table 3. The Diels-Alder reactions were carried out in refluxing toluene or dioxane to give adducts $15-17$ in high yields. Dehydration of the adduct $15 \mathrm{~b}\left(\mathrm{R}=\mathrm{Et}, \mathrm{R}^{\prime}=\mathrm{Ph}\right)$ was examined with various acids including $\mathrm{HBr}, \mathrm{BF}_{3} \mathrm{OEt}_{2}, \mathrm{Sc}(\mathrm{OTf})_{3}$, and $p$-toluenesulfonic acid ( $p$-TsOH). The best yield was observed under conditions with trifluoromethanesulfonic acid. Naphthalene-2,3:6,7-bis (dicarboximide)s 18-20 were obtained as colorless to yellow solids and their structures were confirmed by spectroscopic analysis.<smiles>[R]N1C(=O)c2cc3c([Al])oc([Al])c3cc2C1=O</smiles>

6: $\mathrm{Ar}=\mathrm{Ph}$

7: $\mathrm{Ar}=4-\mathrm{MeC}_{6} \mathrm{H}_{4}$

8: $\mathrm{Ar}=4-\mathrm{MeOC}_{6} \mathrm{H}_{4}$<smiles>[R]N1C(=O)C=CC1=O</smiles>
$\mathrm{R}^{\prime}=\mathrm{Ph}, \mathrm{Me}, \mathrm{Et}$, c-Hex<smiles>[R]N1C(=O)c2cc3c(cc2C1=O)C1(Br)OC3(Br)C2C(=O)N([R])C(=O)C21</smiles>

15: $\mathrm{Ar}=\mathrm{Ph}$

16: $\mathrm{Ar}=4-\mathrm{MeC}_{6} \mathrm{H}_{4}$ 17: $\mathrm{Ar}=4-\mathrm{MeOC}_{6} \mathrm{H}_{4}$

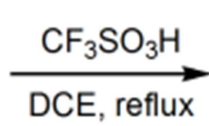<smiles></smiles>

18: $\mathrm{Ar}=\mathrm{Ph}$

19: $\mathrm{Ar}=4-\mathrm{MeC}_{6} \mathrm{H}_{4}$ 20: $\mathrm{Ar}=4-\mathrm{MeOC}_{6} \mathrm{H}_{4}$

Scheme 3. Synthesis of naphthalene-2,3:6,7-bis(dicarboximide)s 18-20.

Table 3. Results of two-step synthesis of naphthalene-2,3:6,7bis(dicarboximide)s 18-20 from isobenzofuranimides 6-8.

\begin{tabular}{lllll}
\hline $\mathbf{A r}$ & $\mathbf{R}$ & R' & product & yield (\%) \\
\hline $\mathrm{Ph}$ & $\mathrm{Me}$ & $c$-hex & $18 \mathrm{a}$ & 87 \\
$\mathrm{Ph}$ & $\mathrm{Et}$ & $\mathrm{Ph}$ & $18 \mathrm{~b}$ & 64 \\
$\mathrm{Ph}$ & $\mathrm{Ph}$ & $\mathrm{Ph}$ & $18 \mathrm{c}$ & 49 \\
$\mathrm{Ph}$ & $4-\mathrm{MeOC}_{6} \mathrm{H}_{4}$ & $\mathrm{Et}$ & $18 \mathrm{~d}$ & 69 \\
$4-\mathrm{MeC}_{6} \mathrm{H}_{4}$ & $\mathrm{Me}$ & $c$-hex & $19 \mathrm{a}$ & 72 \\
$4-\mathrm{MeC}_{6} \mathrm{H}_{4}$ & $\mathrm{Et}$ & $\mathrm{Ph}$ & $19 \mathrm{~b}$ & 68 \\
$4-\mathrm{MeC}_{6} \mathrm{H}_{4}$ & $\mathrm{Ph}$ & $\mathrm{Ph}$ & $19 \mathrm{c}$ & 81 \\
$4-\mathrm{MeC}_{6} \mathrm{H}_{4}$ & $4-\mathrm{MeOC}_{6} \mathrm{H}_{4}$ & $\mathrm{Et}$ & $19 \mathrm{~d}$ & 71 \\
$4-\mathrm{MeOC}_{6} \mathrm{H}_{4}$ & $\mathrm{Me}$ & $c$-hex & $20 \mathrm{a}$ & 84 \\
$4-\mathrm{MeOC}_{6} \mathrm{H}_{4}$ & $4-\mathrm{MeOC}_{6} \mathrm{H}_{4}$ & $\mathrm{Et}$ & $20 \mathrm{~b}$ & 70 \\
\hline
\end{tabular}

Absorption and emission spectra of $18 \mathrm{a}-18 \mathrm{~d}$ and $18 \mathrm{a}-20 \mathrm{a}$ are shown in Figure 2 and 3 and their optical properties are summarized in Table 4. The tailing absorptions of $N$-aryl-substituted bis(dicarboximide)s $18 \mathrm{~b}-\mathrm{d}$ are not depending on their concentrations and hence are thought to be intrinsic dual emission, which has been seen in $N$-phenyl-2,3-naphtalimides [5]. Among them, $N$-p-anisyl- $N$ '-ethyl derivative $18 \mathrm{~d}$ showed clearly the dual bands in the emission spectrum. Emission quantum yields were found a range of $0.01-8 \%$. Comparison of the absorption and emission spectra of $18 \mathrm{a}-20 \mathrm{a}$ indicates an effect of the substituents on the phenyl groups at the 1,4-positions of the naphthalene ring. That is, the long-wavelength absorption of $20 \mathrm{a}$ is slightly different from those of $18 \mathrm{a}-19 \mathrm{a}$ and the emission maximum shows a red shift depending on electron-donating nature of the substituent on the phenyl rings, indicating possibility to control emission wavelength by the substituent.

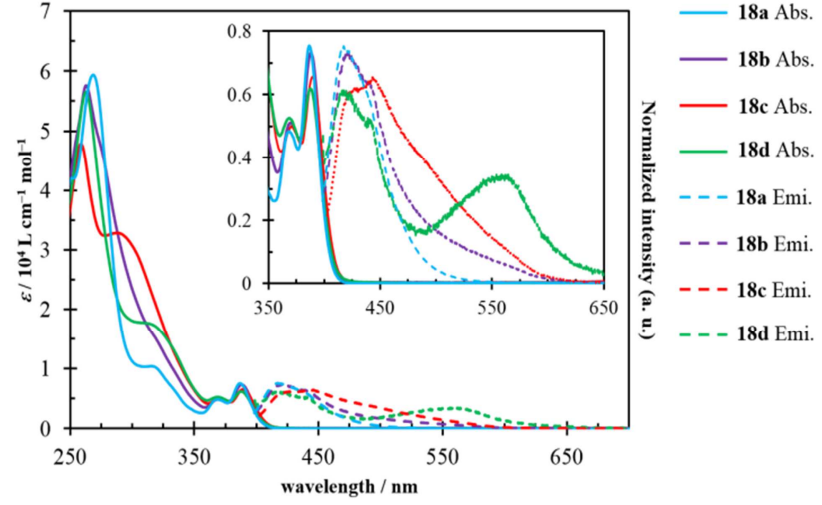

Figure 2. UV-vis absorption (solid line) and emission (broken line) spectra of naphthalene-2,3:6,7-bis(dicarboximide)s 18a-18d.

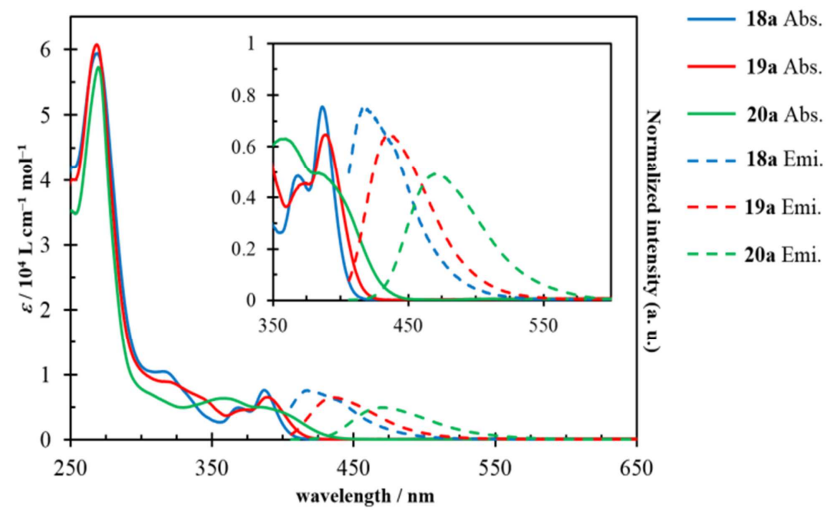

Figure 3. UV-vis absorption (solid line) and emission (broken line) spectra of naphthalene-2,3:6,7-bis(dicarboximide)s 18a-20a. 


\begin{tabular}{|c|c|c|c|c|c|}
\hline imides & $\lambda_{\text {abs }} / \mathbf{n m}$ & $\log \varepsilon$ & $\lambda_{\mathrm{emi}} / \mathbf{n m}$ & Stokes shift / nm & $\Phi / \%$ \\
\hline $18 \mathrm{a}$ & 387 & 3.88 & 417 & 30 & 3 \\
\hline $18 \mathrm{~b}$ & 388 & 3.86 & 421 & 33 & 1 \\
\hline $18 \mathrm{c}$ & 389 & 3.82 & $\begin{array}{l}425 \\
429 \mathrm{sh}\end{array}$ & $\begin{array}{l}36 \\
100\end{array}$ & 0.3 \\
\hline $18 \mathrm{~d}$ & 389 & 3.88 & $\begin{array}{l}418 \\
559\end{array}$ & $\begin{array}{l}29 \\
170\end{array}$ & 0.01 \\
\hline 19a & 389 & 3.81 & 436 & 47 & 4 \\
\hline $20 \mathrm{a}$ & 385 & 3.69 & 470 & 85 & 8 \\
\hline
\end{tabular}

\section{Conclusion}

We have demonstrated synthesis of the novel isobenzofuran-dicarboximides by phosphine-assisted annulation of 2,5-diarylfuran-3,4-dicarbaldehyde with maleimide. These isobenzofurans were transformed into the corresponding naphthalene-2,3:6,7-bis(dicarboximide)s by the Diels-Alder reaction with another maleimide and subsequent dehydration with the aid of trifluoromethanesulfonic acid. The absorption and emission properties are also described. Further study on synthesis of novel arene-dicarboximides by cycloaddition reaction and subsequent dehydration is now actively in progress.

\section{Acknowledgements}

A financial support from the Faculty of Science in Shinshu University (for M.O.) is greatly acknowledged.

\section{References}

[1] Y. Maruoka, "Photoreactions of cyclic imides. Examples of synthetic organic photochemistry", Acc. Chem. Res. 1978, 11, 407-413.

[2] A. Demeter, L. Biczók, T. Bérces, V. Wintgens, P. Valat, and J. Kossanyi, "Laser photolysis studies of transient processes in the photoreduction of naphthalimides by aliphatic amines", $J$. Phys. Chem. 1993, 97, 3217-3224.

[3] K. Maruyama, and Y. Kubo, "Photochemistry of phthalimides with olefins. Solvent-incorporated addition vs. cycloaddtion to imide $\mathrm{C}(=\mathrm{O})-\mathrm{N}$ bond accompanying ring enlargement", J. Org. Chem. 1985, 50, 1426-1435.

[4] Y. Kubo, and M. Suto, T. Arai, P. H. Mazzocchi, L. Klingler, D. Shook, and C. Somich, "Photochemical reactions of $N$-methylnaphthalene-2,3-dicarboximides with alkenes", $J$. Org. Chem. 1986, 51, 4404-4411.

[5] P. Valat, V. Wintgens, J. Kossanyi, L. Biczók, A. Demeter, and T. Bérces, "Influence of geometry on the emitting properties of 2,3-naphthalimides", J. Am. Chem. Soc. 1992, 114, 946-953.

[6] A. Demeter, T. Bérces, L. Biczók, V. Wintgens, P. Valat, and J. Kossanyi, "Comprehensive model of the photophysics of $N$-phenylnaphthalimides: The role of solvent and rotational relaxation", J. Phys. Chem. 1996, 100, 2001-2011.
[7] J. C. Netto-Ferreira, V. Wintgens, L. F. V. Ferreira, A. R. Garcia, L. M. Ilharco, and M. J. Lemos, "Solid complexes between $\mathrm{N}$-(para-alkylphenyl)-2,3-naphthalimides and -cyclodextrin: Characterization by diffuse reflectance infrared Fourier transform spectroscopy and laser induced luminescence studies", J. Photochem. Photobiol. A: Chem. 2000, 132, 209217.

[8] P. Valat, V. Wintgens, J. Kossanyi, L. Biczók, A. Demeter, and T. Bérces, "Temperature-dependent behavior of the dual fluorescence of

2-(3-fluorophenyl)-2,3-dihydro-1 $H$-benzo[f]isoindole-1,3-dion e", Helv. Chim. Acta 2001, 84, 2813-2832.

[9] P. Nandhikonda and M. D. Heagy, "Dual fluorescent $\mathrm{N}$-aryl-2,3-naphthalimides: Applications in ratiometric DNA detection and white organic light-emitting devices", Org. Lett. 2010, 12, 4796

[10] M. Oda, T. Nakamura, M. Neha, D. Miyawaki, A. Ohta, S. Kuroda, and Ryuta Miyatake "A short-step synthesis of 1,6-methanoannulene-3,4-dicarboximides and their benzene-, naphthalene-, and thiophene-annulated compounds", Eur. $J$. Org. Chem. 2014, 5976-5985.

[11] Y. Zhong, B. Kumar, S. Oh, M. T. Trinh, Y. Wu, K. Elbert, P. Li, X. Zhu, S. Xiao, F. Ng, M. L. Steigerwald, and C. Nuckolls, "Helical ribbons for molecular electronics", J. Am. Chem. Soc. 2014, 136, 8122-8130.

[12] X. Zhan, A. Facchetti, S. Barlow, T. J. Marks, M. A. Ratner, M. R. Wasielewski, and S. R. Marder, "Rylene and related diimides for organic electronics", Adv. Mater. 2011, 23, 268284.

[13] S. Guha, F. S. Goodson, L. J. Corson, and S. Saha, "Boundaries of anion/naphthalenediimide interactions; From anioninteractions to anion-induced charge-transfer and electron-transfer phenomena", J. Am. Chem. Soc. 2012, 134, 13679-13691.

[14] H. Cao, V. Chang, R. Hernandez, and M. D. Heagy, "Matrix screening of substituted $N$-aryl-1,8-naphthalimides reveals new dual fluorescent dyes and unusually bright pyridine derivatives", J. Org. Chem. 2005, 71, 4929-4934.

[15] K. Imaizumi, H. Terasima, K. Akasaka, and H. Ohrui, "Highly potent chiral labeling reagents for the discrimination of chiral alcohols", Anal. Sci. 2003, 19, 1243-1249.

[16] L. Kürti and B. Czakó, Strategic Applications of Named Reactions in Organic Synthesis, pp182-184, Elsevier Academic Press, Amsterdat, 2005.

[17] M. Sato, S. Ebine, and S. Akahori, "Condensation of halobenzenes and haloferroces with phthalimide in the presence of copper (I) oxide; A simplified Gabriel reaction", Synthesis 1982, 472-473.

[18] S. Kuroda, N. Tamura, R. Miyatake, N. Matsumoto, Y. Horino, D. Miyawaki and M. Oda, "Synthesis and emission properties of 1,6-methanoannulene-3,4-dicarboximides, Heterocycles 2011, 83, 789-796.

[19] M. P. Cava and P. L. Shirley, "Condensed cyclobutane aromatic compounds. X. Naphtho[b]cyclobutene", J. Am. Chem. Soc. $1960,82,654-656$.

[20] T. Iwanaga, R. Tanaka, and S. Toyota, "Introduction of an arylethynyl group onto an anthracene bisimide core for molecular design of new $\pi$-conjugated compounds", Chem. Lett. 2014, 43, 105-107. 
[21] Y. Nishina, T. Kida, and T. Ureshino, "Facile $\mathrm{Sc}(\mathrm{OTf})_{3}$-catalyzed generation and successive aromatization of isobenzofuran from o-dicarbonylbenzenes", Org. Lett. 2011, 13, 3960-3963.

[22] C. R. Hickenboth, J. S. Moore, S. R. White, N. R. Scott, J. Baudry, and S. R Wilson, "Biasing reaction pathways with mechanical force", Nature 2007, 446, 423-427.

[23] M. J. Haddadin, B. J. Agha, and R. F. Tabri, "Syntheses of some furans and naphtha $[2,3-c]$ derivatives of furan, pyrrole, and thiophene", J. Org. Chem. 1979, 44, 494-497.

[24] M. Oda, H. Shimosasa, Y. Kumai, A. Ohta, and R. Miyatake, "An improved synthesis of arenedicarboximides by phosphine-assisted annulation of arene-1,2-dicarbaldehyde with $N$-substituted maleimide", Modern Chem. 2014, 2(4), 29 35 .

[25] J. Wang, R. Zhou, Z.-R. He, and Z. He, "Phosphane-mediated domino synthesis of tetrasubstituted furans from simple terminal activated olefins", Eur. J. Org. Chem. 2012, 6033604.

[26] TD-DFT calculations for the simplified derivative with a hydrogen atom at the imide nitrogen instead of a phenyl group of $6 \mathrm{a}$ were carried out by with the Gaussian 03 program, Revision C.01 program, Gaussian, Inc.: Pittsburgh, PA, 2003. The result is as follows: For 1st excited state, calculated wavelength is $464 \mathrm{~nm}(2.6721 \mathrm{eV})$, its oscillator strength $f$ is 0.2561 , and the description is HOMO->LUMO (0.62998). For 2nd excited state, calculated wavelength is $404 \mathrm{~nm}(3.0637 \mathrm{eV})$, its oscillator strength $f$ is 0.2641 , and the description is HOMO->LUMO + 1(0.65562).

[27] X.-F. Zhang and X. Li, "The photostability and fluorescence properties of diphenylisobenzofuran", J. Lumines. 2011, 131, 2263-2266.

[28] J. Jacq, S. Tsekhanovich, M. Orio, C. Einhorn, J. Einhorn, B. Bessières, J. Chauvin, D. Jouvenot, and F. Loiseau, "Structure and dynamics of the excited states of 1,3-diarylisobenzofurans: An experimental and theoretical study", Photochem. \& Photobio. 2012, 88, 633-638.

[29] D.-T. Hsu and C.-H. Lin, "Synthesis of benzo[c] and naphtho[ $[c]$ heterocycle diesters and dinitriles via homoelongation," J. Org. Chem. 2009, 74, 9180-9187.

[30] M. P. Cava, A. A. Deana, K. Muth, and m. J. Mitchell, Org. Synth. 1973, Coll. Vol. 5, 944-945.

[31] W. R. Roderick, "The isomerism of $N$-substituted maleimide," J. Am. Chem. Soc. 1957, 79, 1710-1712.

[32] H. Firouzabadi, D. Mohajer, and M. Entezari-Moghadam, "Barium ferrate monohydrate $\mathrm{BaFeO}_{4} \cdot \mathrm{H}_{2} \mathrm{O}$, a useful oxidant for the oxidation of organic compounds under aprotic conditions", Bull. Chem. Soc. Jp. 1988, 61(6), 2185-2189. 